\title{
Dysregulation of microRNA-23b-3p contributes to the development of intracranial aneurysms by targeting phosphatase and tensin homolog
}

\author{
DONG GUO*, YE-WEI WANG* , LEI YAN, JI MA, XIN-WEI HAN and SHAO-FENG SHUI \\ Department of Interventional Radiology, The First Affiliated Hospital of Zhengzhou University, \\ Zhengzhou, Henan 410052, P.R. China
}

Received January 16, 2016; Accepted October 19, 2017

DOI: $10.3892 /$ ijmm.2018.3706

\begin{abstract}
MicroRNA-23b-3p (miR-23b-3p) has been reported to be involved in the pathogenesis of a number of diseases, including non-small cell lung cancer and gastric cancer, by acting on different signaling pathways. The present study aimed to understand the association between the miR-23b-3p level of intracranial aneurysms (IAs) and the mechanism involved. Computational analysis was used to search for the target of miR-23b-3p, and luciferase assay was used to validate the miRNA/target association. Western blot analysis and reverse transcription-quantitative polymerase chain reaction (RT-qPCR) were used to determine the expression of miR-23b-3p and phosphatase and tensin homolog (PTEN), and their expression in smooth muscle cells (SMCs) treated with miRNA mimic or inhibitor. Firstly, an online miRNA database (www.mirdb.org) was searched using the 'seed sequence' located within the 3'-untranslated region of the target gene, and then PTEN was validated as the direct target gene via a luciferase reporter assay system. The negative regulatory association between miR-23b-3p and PTEN was determined through the analysis of the relative luciferase activity. Additionally, RT-qPCR and western blot analysis was performed in order to assess the mRNA and protein expression levels of PTEN among IA $(n=32)$ and control $(n=17)$ groups or cells treated with scramble control, miR-23b-3p mimics, PTEN siRNA and miR-23b-3p inhibitors to verify the negative regulatory association between miR-23b-3p and PTEN. Experiments were then performed to investigate the effect of miR-23b-3p and PTEN on the viability and apoptosis of pulmonary artery SMCs (PASMCs). The
\end{abstract}

Correspondence to: $\mathrm{Dr}$ Shao-Feng Shui, Department of Interventional Radiology, The First Affiliated Hospital of Zhengzhou University, 1 East Jianshe Road, Zhengzhou, Henan 410052, P.R. China

E-mail: iavsmc@163.com

*Contributed equally

Key words: microRNA-23b-3p, intracranial aneurysm, phosphatase and tensin homolog, proliferation, apoptosis results showed that cells transfected with miR-23b-3p inhibitors suppressed the viability of SMCs by promoting the apoptosis of the cells compared with that of the scramble controls, while cells transfected with miR-23b-3p mimics and PTEN siRNA enhanced the viability of VSMCs by inducing apoptosis. This indicated that miR-23b-3p negatively interfered with the viability of the cells, while PTEN positively interfered with the viability of the cells. In conclusion, PTEN was found to be a virtual target of miR-23b-3p, and a negative regulatory association existed between miR-23b-3p and PTEN. miR-23b-3p and PTEN interfered with the viability and apoptosis of SMCs.

\section{Introduction}

Intracranial aneurysms (IAs) are commonly observed in general people with prevalence rate between 2 and $7 \%$ and incidence rate between 1 and 5\%. Subarachnoid bleeding due to a ruptured IA leads to serious consequences resulting in marked morbidity and substantial mortality rates. The mechanisms for the development, progression and collapse of IAs remain largely unknown, although a certain degree of progress has been made recently in their diagnosis and treatment (1). In summary, cerebral infarction has a substantial negative impact on the outcome after SAH. The main potentially treatable factors associated with infarction were symptomatic vasospasm and temperature greater than $38^{\circ} \mathrm{C}$ on day 8 (1). In the clinical setting, systemic high blood pressure is correlated with the initiation of an IA and subarachnoid hemorrhage due to aneurysmal collapse. However, whether high blood pressure causes the initiation of an IA or subarachnoid hemorrhage remains to be confirmed (2-4).

The hypothesis that inflammation is central to IA genesis is strengthened by the flow-mediated dysfunction of the endothelium and the subsequent regulation of smooth muscle cells (SMCs) to a synthetic phenotype (5). The contractile phenotype produces a pro-remodeling and pro-inflammatory milieu, and hence accounts for the inflammation in a number of vascular diseases, which in turn results in persistence and amplification of the recruitment of immune cells and endothelial dysfunction. SMCs of the media progressively transform to a pathophysiological synthetic phenotype from a quiescent contractile phenotype during the development of aneurysms, as observed on sections of human samples for histological examination, and express matrix 
metalloproteinases and pro-inflammatory cytokines $(6,7)$. These proteases have a role in the degradation of the extracellular matrix and in inflammatory regulation, and are accepted to account for the remodeling of IA lesions by the breaking down of the cellular substrate, finally leading to a weakened vascular wall $(8,9)$. A variety of factors are involved in the promotion of SMC apoptosis and increased cell death, and the media of affected cerebral arteries become thinner with the progression of the disease, developing into an aneurysmal cavity (10). This process develops collectively via the apoptosis or degeneration of SMCs, the suppression of the proliferation of SMCs, and the suppression of collagen generation and processing by SMCs, which has been noted in rat IA models (11). However, the major trigger for the remodeling of the media remains unclear.

MicroRNAs (miRNAs/miRs) are an important group of small regulatory RNAs containing $\sim 22$ nucleotides, which are crucial for gene control at the post-transcriptional level. miRNAs bind to the 3'-untranslated regions (3'-UTRs) of mRNAs and either result in message degradation or prevent translation through events mediated by RNA-induced silencing complex (12). This family of small RNAs has been commonly observed since they were first identified $\sim 10$ years ago. Human microRNAs $(n=2,578)$ are listed in the central database and repository of miRNAs in its most recently updated version (v20.0) at miRBase.org (13). miRNAs are located throughout the genome, in polycistronic miRNA clusters consisting of 2-50 miRNAs, with transcriptional units being predominantly intronic to mRNAs.

There are correlations between altered levels of miRNAs and numerous cardiovascular problems complicated with diabetes (14), however, the manner in which diabetes per se regulates the phenotype and function of vascular SMC remains largely unknown. Gene expression alteration and SMC aberrancies in individuals with type II diabetes mellitus can be caused by dysregulated miRNAs triggered by the metabolic milieu (15).

The first miRNA analysis in IA showed reduced levels of 18 miRNAs in IA tissue (16). A total of 681 target genes of the 18 miRNAs were investigated in follow-up studies, including the analysis of the mRNA expression profiles of IA and control samples. There is proliferation of numerous cell types and enrichment in 'migration of phagocytes', as indicated by functional classification of the target genes.

It has previously been demonstrated that miR-23b is differentially expressed in SMCs collected from patients with IA, and PTEN dysregulation has also been indicated to be involved in the molecular mechanism of SMC apoptosis $(16,17)$. By searching the online miRNA database, it was found that PTEN is a virtual target of miR-23b. The present study validated PTEN as a target of miR-23b, and verified the involvement of miR-23b and PTEN in the development of IA. The samples used in the present study were surgically removed aneurysm tissues.

\section{Materials and methods}

Patients and tissue samples. The subjects were unrelated individuals assessed at the Department of Interventional Radiology, The First Affiliated Hospital of Zhengzhou University (Henan, China). For the case group, the trial recruited 32 sporadic intact IA patients (mean age, 48; age range, 39-65), while for the control group (mean age, 45; age range, 35-55), 17 individuals without any health problems were recruited, between July 2013 and September 2014. The patients with IA had been diagnosed by magnetic resonance angiography digital subtraction angiography or three-dimensional computed tomography angiography. The patients provided written signed informed consent for participation in the study, and the study protocol was approved by the Research Ethics Committee at the First Affiliated Hospital of Zhengzhou University.

RNA isolation and reverse transcription-quantitative polymerase chain reaction $(R T-q P C R)$. The total RNA, including miRNA, was isolated from vascular SMCs (VSMCs; isolated from the vessel walls) and extracted using TRIzol reagent (Thermo Fisher Scientific, Inc., Waltham, MA, USA). RNA concentration and purity were determined using a NanoDrop ND-1000 spectrophotometer (Nano Drop Technologies Inc., Rockland, DE, USA), with a 260/280 value $>1.8$ considered acceptable.

RNA was extracted from pulmonary artery SMCs (PASMCs) using TRIzol reagent (Thermo Fisher Scientific, Inc.) and subsequently purified by column with RNeasy kits (Qiagen GmbH, Hilden, Germany). For RT-qPCR of miR-23b-3p, a 7900HT Fast Real-Time PCR system (Applied Biosystems; Thermo Fisher Scientific, Inc.) was used to reverse-transcribe $100 \mathrm{ng}$ total RNA. The reverse transcription kit used was mirVana ${ }^{\mathrm{TM}}$ qRT-PCR miRNA Detection kit (Invitrogen; Thermo Fisher Scientific, Inc.). For RT-qPCR of mRNAs, a High Capacity cDNA Archive kit (Applied Biosystems; Thermo Fisher Scientific, Inc.) was used to synthesize cDNA from $1 \mathrm{mg}$ total RNA. The SYBR Green assay (Applied Biosystems; Thermo Fisher Scientific, Inc.) was used with the Applied Biosystems Real-Time PCR system (Applied Biosystems; Thermo Fisher Scientific, Inc.) to detect the expression of PTEN and miR-23b-3p. The RT primer sequence used was: 5'-GTCGTATCCAGTGCGTGTCGTGG AGTCGGCAATTGCACTGGATACGACGGTAAT-3' (forward, 5'-GGGATCACATTGCCAGGG-3' and reverse, 5'-CAGTGC GTGTCGTGGAGT-3'). Mature sequence hsa-miR-23b-3p was used as reference gene. The thermocycling conditions were as follows: $95^{\circ} \mathrm{C}$ for $10 \mathrm{~min}, 40$ cycles at $95^{\circ} \mathrm{C}$ for $30 \mathrm{sec}$, $60^{\circ} \mathrm{C}$ for $30 \mathrm{sec}$ and $72^{\circ} \mathrm{C}$ for $30 \mathrm{sec}$. The quantification method used was the $2^{-\Delta \Delta \mathrm{Cq}}$ method (18).

Cell culture and transfection. The VSMCs were cultured at $37^{\circ} \mathrm{C}$ in a humidified atmosphere of $5 \% \mathrm{CO}_{2}$, and maintained in Dulbecco's modified Eagle's medium (DMEM), supplemented with $10 \%$ fetal bovine serum, $100 \mathrm{U} / \mathrm{ml}$ penicillin and $100 \mathrm{mg} / \mathrm{ml}$ streptomycin (all from Thermo Fisher Scientific, Inc.). When $80 \%$ confluence was reached, the VSMCs were transfected in suspension into 6-well plates $\left(5-6 \times 10^{5}\right.$ cells/well) using Lipofectamine 2000 (Invitrogen; Thermo Fisher Scientific, Inc.) according to the manufacturer's instructions. miR-23b-3p mimics, inhibitor or PTEN siRNA were used to transfect the cultured cells, and VSMCs transfected with a scramble control were used as a control (transfection reagent, Lipofectamine 2000; concentration, $2 \mu \mathrm{l}$ ). The sequences used were as follows: miR-23b-3p mimics, 5'-ATCACATTGCCAGGGATTACC-3' and miR-23b-3p inhibitor, 5'-GGTAATCCCTGGCAATGTGAT-3'. The growth medium was changed and the total cellular protein or RNA was isolated for further experiments $4 \mathrm{~h}$ later. Western blot analysis and RT-PCR were performed to determine the expression of PTEN and were performed according to the methods stated below. 
Vector construction and mutagenesis. The TargetScan public database (www.targetscan.org) was searched for the target gene of miR-23b-3p, and PTEN was found to be the putative miR-23b-3p binding site. The 3'-UTR of PTEN containing the binding site of miR-23b-3p was amplified through PCR and inserted into the psiCHECK-2 reporter vector (Promega Corporation, Madison, WI, USA). PCR was performed according to the methods already stated. Meanwhile, mutagenesis was performed by replacing the miR-23b-3p binding site nucleotides and introduced into the control vector (Ambion; Thermo Fisher Scientific, Inc.).

Cell proliferation assay. The effects of miR-23b-3p overexpression on VSMC proliferation were assessed using Cell Counting Kit-8 (CCK-8; Dojindo Molecular Technologies, Inc., Kumamoto, Japan). The PASMC proliferation reagent WST-1 was used to quantify proliferation rates of PASMCs. Briefly, PASMCs $\left(0.5 \times 10^{6}\right.$ cells/well $)$ were transferred to 96 -trawell plates and AZD5438, NU6140, and CPT were used to treated the proliferating cells in serum containing medium. Following treatment, the PASMCs were washed in DMEM without L-glutamine and phenol red. WST-1 reagent was diluted (1:10 final dilution) in DMEM without phenol red and with L-glutamine (all from Sigma-Aldrich; Merck KGaA, Darmstadt, Germany). This diluted tetrazolium WST-1 reagent $(200 \mu 1)$ was then added to each well and absorption was measured at 2, 4 and $6 \mathrm{~h}$. Diluted WST-1 reagent was used for the background control. Absorbance was read at $440 \mathrm{~nm}$ using an ELISA plate reader.

Luciferase assay. The 3'-UTR of PTEN was cloned into the psiCHECK-2 vector, between the XhoI/NotI site, immediately downstream of the firefly luciferase gene in the pGL3-control vector (both Promega Corporation). For selected targets, inverse PCR with non-overlapping primers carrying the mutated sequences was used to introduce 3 point mutations into the 7-nt seed-binding sequence (19). Lipofectamine 2000 (Invitrogen Life Technologies) was used to co-transfect the $10 \mathrm{ng}$ of each psi-CHECK-2 construct and $10 \mathrm{nM}$ miRNA duplexes or into VSMCs in a 96-well plate. The cell extract was harvested 2 days later and assayed using the Dual-Luciferase reporter system (Promega Corporation) to measure Firefly and Renilla luciferase activities according to the manufacturer's protocols. Each experiment was analyzed in triplicate in three independent experiments.

Western blot analysis. Ice-cold PBS and radio immunoprecipitation assay lysis buffer were used to wash the cell monolayers in ice cold extraction buffer $[20 \mathrm{mM}$ Tris- $\mathrm{HCl}$ (pH 7.5), 1 mM Na${ }_{2}$ EDTA, $150 \mathrm{mM} \mathrm{NaCl}, 1 \%$ Triton X-100, $1 \mathrm{mM}$ EGTA, $2.5 \mathrm{mM}$ sodium pyrophosphate, $1 \mathrm{mM} \mathrm{Na} \mathrm{VO}_{4}$ and $1 \mathrm{mM}$-glycerophosphate; Thermo Fisher Scientific, Inc.] containing protease inhibitor cocktail (Sigma-Aldrich; Merck $\mathrm{KGaA}$ ) for $10 \mathrm{~min}$ according to the manufacturer's protocols. Cell lysates were centrifuged at $16,770 \mathrm{x} \mathrm{g}$ at $4^{\circ} \mathrm{C}$ for $10 \mathrm{~min}$ and proteins were separated by $12 \%$ SDS-PAGE. The target proteins were transferred to Hybond ECL nitrocellulose membrane (GE Healthcare, Chicago, IL, USA), blocked using 5\% skimmed dry milk and $0.1 \%$ Tween-20 for $2 \mathrm{~h}$ at room temperature and detected with the indicated antibodies [primary antibody, PTEN (cat. no. 9552S; dilution, 1:1,000; Cell Signaling Technology, Inc., Danvers, MA, USA) at $4^{\circ} \mathrm{C}$ for

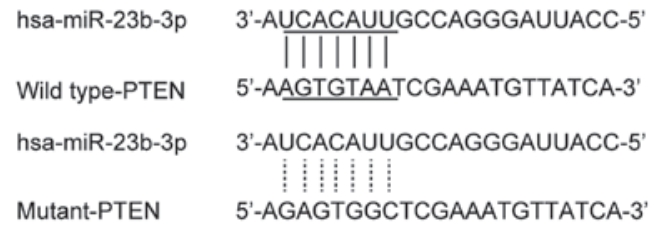

Figure 1. PTEN as the candidate target gene of miR-23b-3p in cells with the 'seed sequence' in the 3'-untranslated region. PTEN, phosphatase and tensin homolog; miR, microRNA.

12 h; secondary antibody, HRP-linked antibody (cat. no. 7075S; dilution, 1:3,000; Cell Signaling Technology, Inc.) at $25^{\circ} \mathrm{C}$ for $2 \mathrm{~h}$ ] in TBS buffer (5\% skimmed dry milk and $0.1 \%$ Tween-20; blotting grade; Bio-Rad Laboratories, Inc., Hercules, CA, USA). The protein determination method was BCA assay (Beyotime Institute of Biotechnology, Haimen, China). Next, horseradish peroxidase-conjugated secondary antibodies were used for $1 \mathrm{~h}$ at room temperature according to the manufacturer's instructions. The visualization reagent used was Amersham ECL Prime Western Blotting Detection reagent (GE Healthcare).

Apoptosis analysis. Annexin V/propidium iodide staining with the apoptosis detection kit (Nanjing KeyGen Biotech Co., Ltd., Nanjing, China) was used to detect VSMC apoptosis. To measure the apoptosis of VSMCs, flow cytometry was performed with Annexin V-FITC (fluorescein isothiocyanate)/PI Apoptosis Detection kit (Sigma-Aldrich; Merck KGaA). VSMCs were harvested following the transfection with miR-23b-3p mimic after $48 \mathrm{~h}$, and then washed twice using PBS. Cells were resuspended at a final density of $4 \times 10^{5}$ cells per well with $1 \mathrm{X}$ binding buffer. After treated with $5 \mu \mathrm{l} \mathrm{PE}$ Annexin V and $5 \mu \mathrm{l}$ 7-AAD, $100 \mu \mathrm{l}$ cell suspension was incubated in darkness for $15 \mathrm{~min}$. FACS (BD Biosciences; San Jose, CA, USA) was used to detect cell apoptosis. The $\mathrm{y}$-axis and $\mathrm{x}$-axis were used to show plasma membrane integrity and Annexin V immune fluorescence, respectively. Assays were performed in three independent experiments, and the ratio of dead cells/total cells was analyzed statistically.

Statistical analysis. All data are presented as the mean \pm standard deviation. Each experiment was analyzed for three times, with three samples for each. The results of western blot analyses from three experiments are shown. One-way analysis of variance and Student-Newman-Keuls post hoc test determined the significance of the differences between the means. $\mathrm{P}<0.05$ was considered to indicate a statistically significance.

\section{Results}

PTEN is a virtual target of miR-23b-3p. miR-23b-3p has been reported to be involved in numerous diseases (20), including non-small cell lung cancer and gastric cancer, by acting on different signaling pathways. The present study aimed to increase our understanding of the association between miR-23b-3p level and IA. Online miRNA target prediction tools were used to search for the regulatory gene of miR-23b-3p, and consequently PTEN was identified in cells with the 'seed sequence' in the 3'-UTR (Fig. 1). A luciferase activity reporter assay was conducted in the VSMCs to determine the interactions, and only the luciferase activity from the miR-23b-3p 


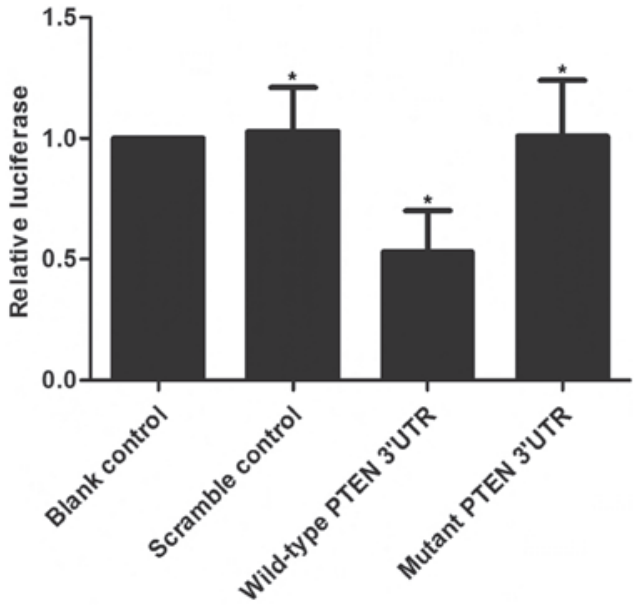

Figure 2. Luciferase activity reporter assay was conducted to verify PTEN as the direct target gene of miR-23b-3p. PTEN, phosphatase and tensin homolog; miR, microRNA; UTR, untraslated region. ${ }^{\text {P }}<0.05$ vs. control.

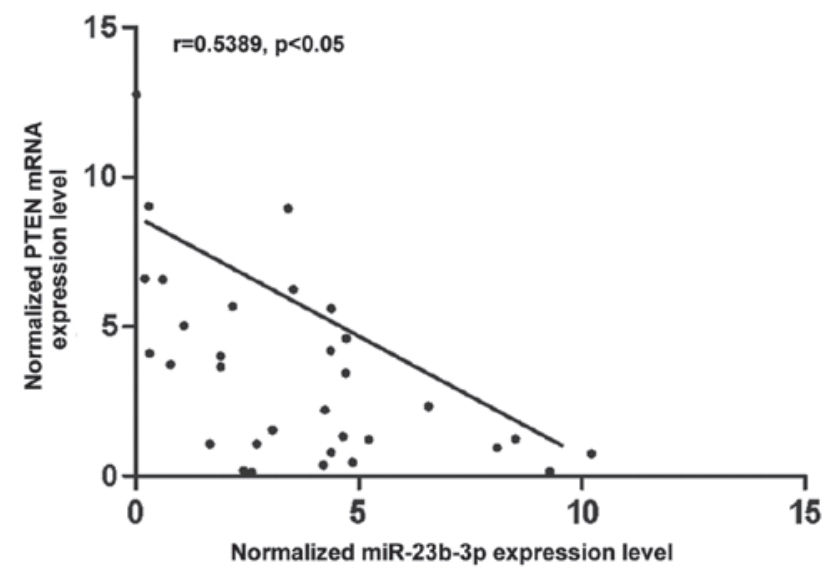

Figure 3. Correlation (Pearson correation analysis) between the expression level of miR-23b-3p and PTEN mRNA between patients (intracranial aneurysm, $n=32$; control, $n=17$ ). PTEN, phosphatase and tensin homolog; miR, microRNA.

and wild-type PTEN 3'-UTR cotransfected cells decreased significantly (Fig. 2). The activity of cells with miR-23b-3p and mutant PTEN 3'-UTR cotransfection was similar to that of the scramble control (Fig. 2). The results demonstrated that PTEN was a validated target of miR-23b-3p in the VSMCs. To further understand the interactions between miR-23b-3p and PTEN, the correlation between the expression level of miR-23b-3p and PTEN mRNA among the tissues was analyzed $(\mathrm{n}=32)$, and a negative regulatory correlation was found (Fig. 3).

Determination of expression patterns of $m i R-23 b-3 p$ and PTEN in tissues of different groups. The tissues of two different groups (IA, $n=32$; control, $n=17$ ) were used to further investigate the impact on the interaction between miR-23b-3p and the PTEN 3'-UTR. qPCR was performed to detect miR-23b-3p and PTEN mRNA expression, with results revealing that compared with that of the normal control, miR-23b-3p expression was decreased in the IA group (Fig. 4A). PTEN mRNA expression (Fig. 4B) was increased in the IA group compared with the normal control, as was PTEN protein expression, according to densitometry analysis (Fig. 4C). To further
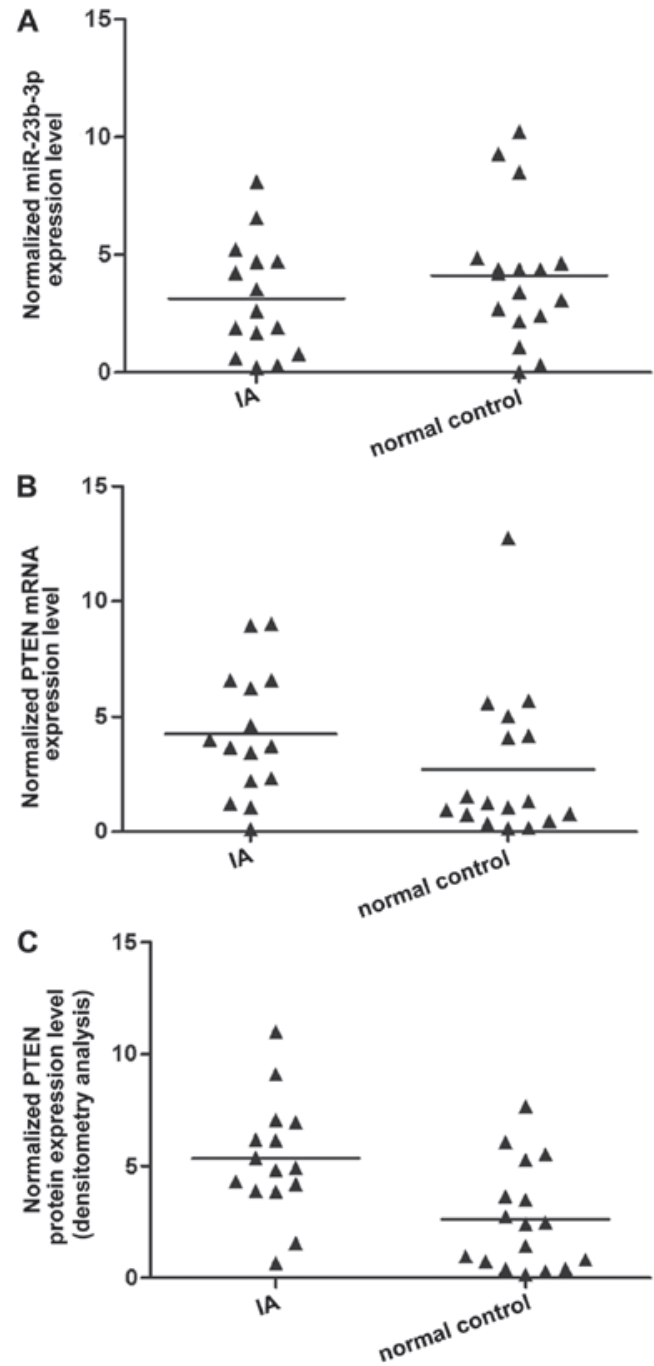

Figure 4. (A) Expression of miR-23b-3p decreased in the IA group compared with that in the normal control group, while the expression of PTEN (B) mRNA and (C) protein increased in the IA group compared with that in the normal group. PTEN, phosphatase and tensin homolog; miR, microRNA; IA, intracranial aneurysm.

validate the hypothesis of the negative regulatory association between miR-23b-3p and PTEN, PTEN mRNA expression in SMCs was investigated, with the SMCs being transfected with scramble control, miR-23b-3p mimics, PTEN siRNA and miR-23b-3p inhibitors. As shown in Fig. 5, the PTEN mRNA expression level of SMCs treated with miR-23b-3p mimics and PTEN siRNA were markedly lower than those of the scramble control, while the expression of cells treated with miR-23b-3p inhibitors was markedly higher than that of the scramble control, validating the negative regulatory association between miR-23b-3p and PTEN.

Alternations in miR-23b-3p and PTEN affect the viability of SMCs. VSMCs were transfected with scramble control, miR-23b-3p mimics, PTEN siRNA and miR-23b-3p inhibitors to evaluate the effect of alternations in miR-23b-3p on the relative viability and apoptosis of the cells. miR-23b-3p inhibitor transfection suppressed the viability of the SMCs (Fig. 6A) by promoting their apoptosis (Fig. 6B) when compared with the scramble controls, while miR-23b-3p mimic and PTEN siRNA transfection enhanced the viability of the VSMCs 
A
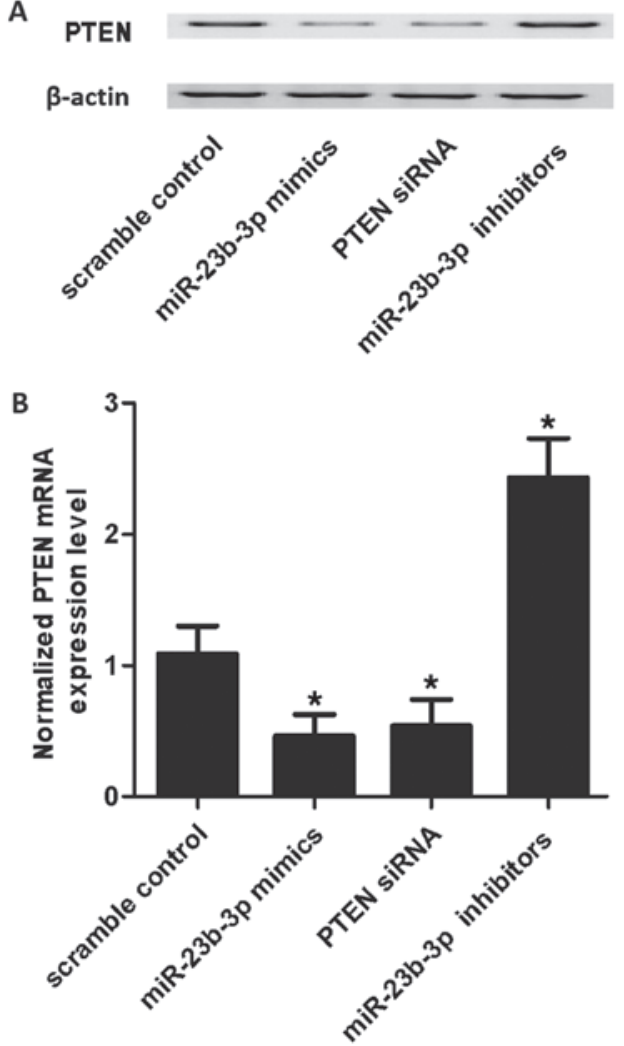

Figure 5. (A and B) SMCs were transfected with scramble control, miR-23b-3p mimics, PTEN siRNA and miR-23b-3p inhibitors. The expression level of PTEN mRNA in the cells treated with miR-23b-3p mimics and PTEN siRNA decreased, while that in cells treated with miR-23b-3p inhibitors increased. PTEN, phosphatase and tensin homolog; miR, microRNA; siRNA, small interfering RNA; SMCs, smooth muscle cells. "P<0.05 vs. control.

(Fig. 6A) by inducing apoptosis (Fig. 6B). This indicates that miR-23b-3p negatively affects the viability of SMCs, while PTEN positively affects the viability of cells.

\section{Discussion}

miR-23b-3p is a member of the miR-23b/27b/24-1 cluster, the dysregulation of which has been demonstrated in a variety of cancer types. In human colon cancer, downregulation of miR-23b-3p is observed, regulating MAP3k1 or FZD7 to trigger the multiple processes of metastasis in vivo. Moreover, miR-23b-3p acts as an oncogenic miRNA to suppress PTEN in renal cell carcinoma (21). Also, thymic lymphoma induced by radiation has been demonstrated to exhibit upregulated expression of miR-23b-3p (22). miR-23b-3p was also found to induce the sensitivity of GC cells to chemotherapy (23). Furthermore, our previous study showed that miR-23b-3p has two directly functional targets, including high-mobility group box 2 and autophagy-related gene 12, which are overexpressed in GC multidrug-resistant cells and positively associated with either chemoresistance or autophagy (24). It has been previously shown that miR-23b is differentially expressed in SMCs collected from patients diagnosed with IA (16). In the present study, tissues were collected from patients with IA $(n=32)$ or unaffected controls $(n=17)$, and $q P C R$ was used to determine the expression of miR-23b-3p. This revealed that the miRNA was substantially downregulated in the IA samples. Furthermore, it
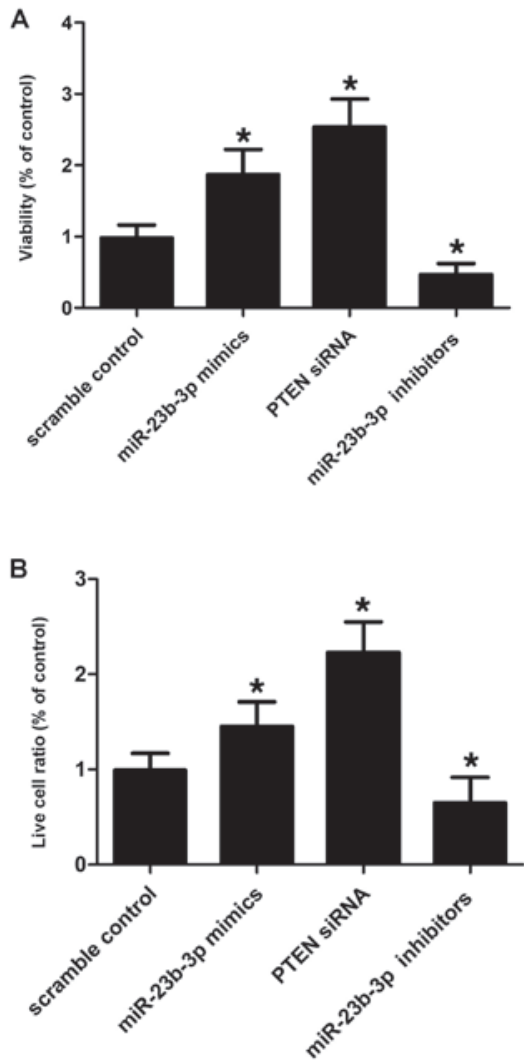

Figure 6. (A and B) SMCs transfected with miR-23b-3p inhibitors showed evidently upregulated viability, while those transfected with miR-23b-3p mimics and PTEN siRNA showed comparably lower viability; SMC cells transfected with miR-23b-3p mimics and PTEN siRNA exhibited decreased apoptosis while those transfected with miR-23b-3p exhibited increased apoptosis. PTEN, phosphatase and tensin homolog; miR, microRNA; siRNA, small interfering RNA; SMCs, smooth muscle cells. ${ }^{*}<<0.05$ vs. control.

was found that PTEN is a virtual target of miR-23b by using an online miRNA database.

In another study, we previously demonstrated that there was an association between miR-23b-3p level and survival in patients with renal carcinoma (22). As with miR-21, all patients exhibited low or moderate expression of miR-23b-3p, but only half of the patients with high miR-23b-3p expression achieved 5-year survival following surgery. miR-23b-3p-knockdown in renal cancer cells contributed to an elevated quantity of apoptotic cells, and reduced cell migration and invasion. Tumor inhibitor PTEN was not detected in tumor samples with high expression of miR-23b-3p and was augmented in miR-23b-3p-knockdown cells. miR-23b-3p directly targeted the 3'-UTR of PTEN, demonstrating that PTEN is involved in the apoptotic mechanism induced by miR-23b-3p. GEN treatment reduced the expression of $\operatorname{miR}-23 b-3 p$, revealing that downregulated miR-23b-3p expression could trigger the pro-apoptotic role of GEN on renal cancer cells (22). In the present study, a luciferase activity reporter assay was conducted in the VSMCs, and it was revealed that only the luciferase activity from the miR-23b-3p and wild-type PTEN 3'-UTR cotransfected cells significantly decreased (Fig. 2), while cells with miR-23b-3p and mutant PTEN 3'-UTR cotransfection showed results similar to that of the scramble control (Fig. 2). This supported PTEN as a validated target of miR-23b-3p in VSMCs. 
PTEN is a lipid phosphatase and protein that serves an inhibitory role in numerous pathways implicated in proliferation, cell survival and inflammation (25). The effect of PTEN has been well established in cancer, but its effects in vascular disorders are less well characterized. Several studies have demonstrated that the inactivation of PTEN contributes to elevated SMC proliferation and reduced cell death $(26,27)$. In addition, vascular injury accounts for inactivation of SMC PTEN and is correlated with elevated formation of neointima (28). SMC-specific-knockout of PTEN in vivo leads to a larger neointima responding to injury of the vessel wall caused by recruitment of inflammatory cells and elevated proliferation (29). An inflammatory SMC phenotype can be promoted by in vitro shRNA-dependent depletion of PTEN, characterized by elevated production of chemokines and inflammatory cytokines, reduced SM-gene expression and elevated proliferation (17). Moreover, a study by Ji et al revealed that miR-21 directly targets PTEN and that miRs such as miR-21 are differentially expressed by SMC when vascular injury occurs (30). In addition, neointima formation is regulated by altered miR-21 in vivo.

In humans, germline mutations of PTEN are correlated with Lhermitte-Duclos disease, Bannayan-Zonana syndrome and Cowden disease $(31,32)$. Patients have common pathological characteristics, such as benign hyperplasias or tumors of different tissue origins, and initiation of hamartomas. A variety of tumors and hyperplasias are developed in mice that are heterozygous for Pten genes during their lifetime (33). Notably, Freeman et al showed that Pten ${ }^{\Delta 5 /+}$ mice exhibited vascular abnormalities resembling hemangiomas or angiomatosis, and that the genetic background was the basis of the initiation and spectrum of tumor development (34). Moreover, vascular malformation is observed in transgenic murine models with maintained activation of Akt. Abnormal blood vessel formation can be caused by expression of myristoylated-Akt1 in endothelial cells $(35,36)$. It is unclear why endothelial cells are particularly prone to tumor development correlated with loss of three of the four Pten alleles, and this therefore requires further study in ptena ${ }^{+/}$pten $^{-/-}$and ptena $^{-/-}$ptenb ${ }^{+/-}$fish. In the present study, SMCs were transfected with scramble control, miR-23b-3p mimics, PTEN siRNA and miR-23b-3p inhibitors to evaluate the effect of alternation of miR-23b-3p on relative cell viability and apoptosis. It was found that cells transfected with miR-23b-3p inhibitors suppressed the viability of SMCs by promoting the apoptosis of the cells when compared with the scramble controls, while cells transfected with miR-23b-3p mimics and PTEN siRNA enhanced the viability of VSMCs by inducing apoptosis, indicating that miR-23b-3p negatively affected the viability of the cells, while PTEN positively affected the viability of the cells.

In conclusion, PTEN was virtual target of miR-23b-3p and a negative regulatory association existed between miR-23b-3p and PTEN in the present study. miR-23b-3p and PTEN affected the viability and apoptosis of SMCs.

\section{Competing interests}

Authors declare they have no competing interest.

\section{References}

1. Fergusen S and Macdonald RL: Predictors of cerebral infarction in patients with aneurysmal subarachnoid hemorrhage. Neurosurgery 60: 658-667, discussion 667, 2007.

2. Schievink WI: Intracranial aneurysms. N Engl J Med 336: 28-40, 1997.

3. Connolly ES Jr, Choudhri TF, Mack WJ, Mocco J, Spinks TJ, Slosberg J, Lin T, Huang J and Solomon RA: Influence of smoking, hypertension, and sex on the phenotypic expression of familial intracranial aneurysms in siblings. Neurosurgery 48: 64-68, discussion 68-69, 2001.

4. Bonita R: Cigarette smoking, hypertension and the risk of subarachnoid hemorrhage: a population-based case-control study. Stroke 17: 831-835, 1986.

5. Kaźmierski M, Michalewska-Włudarczyk A and Krzych LJ: Intima-media thickness and flow-mediated dilatation in the diagnosis of coronary artery disease in perimenopausal women. Pol Arch Med Wewn 120: 181-188, 2010.

6. Nakajima N, Nagahiro S, Sano T, Satomi J and Satoh K: Phenotypic modulation of smooth muscle cells in human cerebral aneurysmal walls. Acta Neuropathol 100: 475-480, 2000.

7. Aoki T, Kataoka H, Morimoto M, Nozaki K and Hashimoto N: Macrophage-derived matrix metalloproteinase-2 and -9 promote the progression of cerebral aneurysms in rats. Stroke 38: 162-169, 2007.

8. Page-McCaw A, Ewald AJ and Werb Z: Matrix metalloproteinases and the regulation of tissue remodelling. Nat Rev Mol Cell Biol 8: 221-233, 2007.

9. Parks WC, Wilson CL and López-Boado YS: Matrix metalloproteinases as modulators of inflammation and innate immunity. Nat Rev Immunol 4: 617-629, 2004.

10. Meng H, Metaxa E, Gao L, Liaw N, Natarajan SK, Swartz DD, Siddiqui AH, Kolega $\mathrm{J}$ and Mocco J: Progressive aneurysm development following hemodynamic insult. J Neurosurg 114: 1095-1103, 2011.

11. Aoki T, Kataoka H, Ishibashi R, Nozaki K, Morishita R and Hashimoto N: Reduced collagen biosynthesis is the hallmark of cerebral aneurysm: contribution of interleukin-1beta and nuclear factor-kappaB. Arterioscler Thromb Vasc Biol 29: 1080-1086, 2009.

12. Bartel DP: MicroRNAs: genomics, biogenesis, mechanism, and function. Cell 116: 281-297, 2004.

13. Griffiths-Jones S: The microRNA registry. Nucleic Acids Res 32: D109-D111, 2004.

14. Pandey AK, Agarwal P, Kaur K and Datta M: MicroRNAs in diabetes: tiny players in big disease. Cell Physiol Biochem 23: 221-232, 2009.

15. Porter KE and Riches K: The vascular smooth muscle cell: a therapeutic target in type 2 diabetes? Clin Sci (Lond) 125: 167-182, 2013.

16. Jiang Y, Zhang M, He H, Chen J, Zeng H, Li J and Duan R: MicroRNA/mRNA profiling and regulatory network of intracranial aneurysm. BMC Med Genomics 6: 36, 2013.

17. Furgeson SB, Simpson PA, Park I, Vanputten V, Horita H, Kontos CD, Nemenoff RA and Weiser-Evans MC: Inactivation of the tumour suppressor, PTEN, in smooth muscle promotes a pro-inflammatory phenotype and enhances neointima formation. Cardiovasc Res 86: 274-282, 2010

18. Livak and Schmittgen: Analysis of relative gene expression data using real-time quantitative PCR and the 2(-Delta Delta C(T)) Method. Methods 25: 402-408, 2001.

19. Hartl DL and Ochman H: Inverse polymerase chain reaction. Methods Mol Biol 58: 293-301, 1993.

20. Chen D, Wu X, Xia M, Wu F, Ding J, Jiao Y, Zhan Q and An F: Upregulated exosomic miR-23b-3p plays regulatory roles in the progression of pancreatic cancer. Oncol Rep 38: 2182-2188, 2017.

21. Zhang H, Hao Y, Yang J, Zhou Y, Li J, Yin S, Sun C, Ma M, Huang Y and Xi JJ: Genome-wide functional screening of miR-23b as a pleiotropic modulator suppressing cancer metastasis. Nat Commun 2: 554, 2011.

22. Zaman MS, Thamminana S, Shahryari V, Chiyomaru T, Deng G, Saini S, Majid S, Fukuhara S, Chang I, Arora S, et al: Inhibition of PTEN gene expression by oncogenic miR-23b-3p in renal cancer. PLoS One 7: e50203, 2012.

23. Li B, Sun M, Gao F, Liu W, Yang Y, Liu H, Cheng Y, Liu C and Cai J: Up-regulated expression of $\mathrm{miR}-23 \mathrm{a} / \mathrm{b}$ targeted the pro-apoptotic Fas in radiation-induced thymic lymphoma. Cell Physiol Biochem 32: 1729-1740, 2013. 
24. An Y, Zhang Z, Shang Y, Jiang X, Dong J, Yu P, Nie Y and Zhao Q: $\mathrm{miR}-23 \mathrm{~b}-3 \mathrm{p}$ regulates the chemoresistance of gastric cancer cells by targeting ATG12 and HMGB2. Cell Death Dis 6 : e1766, 2015.

25. Oudit GY, Sun H, Kerfant BG, Crackower MA, Penninger JM and Backx PH: The role of phosphoinositide-3 kinase and PTEN in cardiovascular physiology and disease. J Mol Cell Cardiol 37: 449-471, 2004

26. Huang J, Niu XL, Pippen AM, Annex BH and Kontos CD: Adenovirus-mediated intraarterial delivery of PTEN inhibits neointimal hyperplasia. Arterioscler Thromb Vasc Biol 25: 354-358, 2005.

27. Mitra AK, Jia G, Gangahar DM and Agrawal DK: Temporal PTEN inactivation causes proliferation of saphenous vein smooth muscle cells of human CABG conduits. J Cell Mol Med 13: 177-187, 2009.

28. Mourani PM, Garl PJ, Wenzlau JM, Carpenter TC, Stenmark KR and Weiser-Evans MC: Unique, highly proliferative growth phenotype expressed by embryonic and neointimal smooth muscle cells is driven by constitutive Akt, mTOR, and p70S6K signaling and is actively repressed by PTEN. Circulation 109: 1299-1306, 2004.

29. Nemenoff RA, Horita H, Ostriker AC, Furgeson SB, Simpson PA, VanPutten V, Crossno J, Offermanns S and Weiser-Evans MC: SDF-1 $\alpha$ induction in mature smooth muscle cells by inactivation of PTEN is a critical mediator of exacerbated injury-induced neointima formation. Arterioscler Thromb Vasc Biol 31: 1300-1308, 2011.

30. Ji R, Cheng Y, Yue J, Yang J, Liu X, Chen H, Dean DB and Zhang C: MicroRNA expression signature and antisense-mediated depletion reveal an essential role of microRNA in vascular neointimal lesion formation. Circ Res 100: 1579-1588, 2007.
31. Nelen MR, van Staveren WC, Peeters EA, Hassel MB, Gorlin RJ, Hamm H, Lindboe CF, Fryns JP, Sijmons RH, Woods DG, et al: Germline mutations in the PTEN/MMACl gene in patients with Cowden disease. Hum Mol Genet 6: 1383-1387, 1997.

32. Marsh DJ, Dahia PL, Zheng Z, Liaw D, Parsons R, Gorlin RJ and Eng C: Germline mutations in PTEN are present in Bannayan-Zonana syndrome. Nat Genet 16: 333-334, 1997.

33. Suzuki A, de la Pompa JL, Stambolic V, Elia AJ, Sasaki T, del Barco Barrantes I, Ho A, Wakeham A, Itie A, Khoo W, et al: High cancer susceptibility and embryonic lethality associated with mutation of the PTEN tumor suppressor gene in mice. Curr Biol 8: 1169-1178, 1998.

34. Freeman D, Lesche R, Kertesz N, Wang S, Li G, Gao J, Groszer M, Martinez-Diaz H, Rozengurt N, Thomas G, et al: Genetic background controls tumor development in PTEN-deficient mice. Cancer Res 66: 6492-6496, 2006.

35. Sun JF, Phung T, Shiojima I, Felske T, Upalakalin JN, Feng D, Kornaga T, Dor T, Dvorak AM, Walsh K, et al: Microvascular patterning is controlled by fine-tuning the Akt signal. Proc Natl Acad Sci USA 102: 128-133, 2005.

36. Phung TL, Ziv K, Dabydeen D, Eyiah-Mensah G, Riveros M, Perruzzi C, Sun J, Monahan-Earley RA, Shiojima I, Nagy JA, et al: Pathological angiogenesis is induced by sustained Akt signaling and inhibited by rapamycin. Cancer Cell 10: 159-170, 2006. 